\title{
线粒体菼光探针研究新进展
}

\author{
李杨洁吕子奇刘敏邢国文* \\ (北京师范大学化学学院 北京 100875)
}

\begin{abstract}
摘要 近年来，许多基于有机小分子既能定位于线粒体又能够检测线粒体内各类活性小分子的双功能线粒体荧光探针 被相继报道，成功地实现了线粒体内多种活性物种的检测和可视化成像，这些物种包括活性氧、还原性物种、金属离 子、质子、阴离子等. 为进一步对线粒体内特定活性小分子进行组织及活体内无创检测与成像，性能优良的双光子及 近红外探针的设计合成备受瞩目. 围绕近年来出现的线粒体荧光探针依据检测对象进行总结与评述, 展望了线粒体荧 光探针在癌症等病症的前期诊断和后期治疗方面的发展趋势.
\end{abstract}

关键词 线粒体; 苂光探针; 活性小分子; 分子成像

\section{Recent Progresses on Mitochondria-Targetable Fluorescent Probes}

\author{
Li, Yangjie Lü, Ziqi $\quad$ Liu, Min $\quad$ Xing, Guowen* \\ (College of Chemistry, Beijing Normal University, Beijing 100875)
}

\begin{abstract}
Over the past few years, many bifunctional fluorescent probes based on small organic molecules, which can be located on the mitochondria to detect specific reactive small molecules (RSMs), including reactive oxygen species, reducing species, metal ions, proton and small anions, have been reported to be successful in achieving visual imaging in mitochondria. For further detection and imaging of the reactive small molecules in tissue, the design and synthesis of two-photon fluorescent probes and near-infrared fluorescent probes bearing good optical properties become increasingly popular. The recently reported mitochondrial fluorescent probes are classified, summarized and reviewed. The bright prospects of these probes in the application of preliminary diagnosis and therapy of some diseases are also discussed.
\end{abstract}

Keywords mitochondria; fluorescent probes; reactive small molecules; molecular imaging

随着生物科学与技术的不断发展, 人们逐渐从细胞 层面过渡到亚细胞层面认识和研究生命活动的本质. 线 粒体作为细胞内关键的细胞器, 不仅具有为细胞供给能 量、参与代谢等重要功能, 而且参与细胞信号转导和细 胞调亡等重要的生物过程. 大量研究表明, 线粒体的数 量、分布、结构与功能变化等与神经退行性病变(如阿 尔兹海默症、帕金森症)、代谢型疾病(如肥胖症、II 型 糖尿病)、心血管疾病及癌症等病症关系密切. 线粒体被 冠以 “细胞信号传导细胞器” 和 “细胞死亡之马达” 等 称号, 与之相关的 “线粒体学” 已经成为生命科学和医 学等领域的研究热点 ${ }^{[1]}$.

线粒体内活性小分子 (reactive small molecules, RSMs) 作为信号载体参与线粒体内的生物化学反应, 它
们的浓度和时空分布能够影响细胞乃至生物体的多种 生理过程. 因此, 开展线粒体内 RSMs 的相关研究对深 入揭示生物体生命活动规律起着至关重要的作用, 愈来 愈受到研究者们的青睐. RSMs 包括活性氧物种 $[R O S$, Reactive Oxygen Species, 比如次氯酸根 $\left(\mathrm{ClO}^{-}\right)$、过氧亚 硝基阴离子 $\left(\mathrm{ONOO}^{-}\right)$、羟基自由基 $(\cdot \mathrm{OH})$ 、过氧化氢 $\left(\mathrm{H}_{2} \mathrm{O}_{2}\right)$ 、一氧化氮 $(\mathrm{NO})$ 、超氧阴离子自由基 $\left(\mathrm{O}_{2}^{--}\right)$等 $]$; 还 原性物种 $\left[\right.$ 如硫化氢 $\left(\mathrm{H}_{2} \mathrm{~S}\right)$ 、谷胱甘肽 $(\mathrm{GSH})$ 在内的硫醇类 化合物等]; 金属阳离子 $\left(\mathrm{Hg}^{2+} 、 \mathrm{Zn}^{2+} 、 \mathrm{Cu}^{+}\right.$等 $)$、质子 $\left(\mathrm{H}^{+}\right)$、 阴离子 (如 $\mathrm{F}^{-}$)等.

荧光探针有灵敏度高、检测限低、操作方便、生物 成像性能好等优点, 现已成为生命科学研究常用的检测 手段之一 ${ }^{[2]}$. 为了可视化观测线粒体内 RSMs 的时空分

\footnotetext{
* E-mail: gwxing@bnu.edu.cn

Received October 12, 2015; revised December 28, 2015; published online January 7, 2016.

Project supported by the National Natural Science Foundation of China (No. 21272027).

国家自然科学基金(No. 21272027)资助项目.
} 
布, 研究者们设计了一系列基于有机小分子的线粒体靶 向荧光探针. 利用这些探针作为研究工具实现了细胞、 组织和生物体内线粒体 RSMs 的分子成像, 在一定程度 上可视化地监测到了特定 RSMs 在线粒体中的时空分 布. 一般而言, 在线粒体内膜的跨膜电势驱动下, 以三 苯基鏻离子(TPP)或季铵盐阳离子作为定位基团的苂光 探针能够精确定位于线粒体. 另外, 近年来中性线粒体 苂光探针的研究 ${ }^{[3 \sim 8]}$ 也开始涌现.

为了实现线粒体内 RSMs 的有效检测, 荧光探针的 设计应满足化学选择性强、生物兼容性好、生物正交性 高等特点 ${ }^{[9]}$. 与荧光淬灭型探针相比, 荧光增强型探针 更容易设计成为高信噪比、选择性高的探针 ${ }^{[10]}$. 探针结 构中除了探针分子中连接线粒体定位基团外, 识别基团 通过连接臂与荧光团相连, 常基于分子内电荷转移 (ICT)、光诱导电子转移(PET)、荧光共振能量转移 (FRET)、聚集诱导发光(AIE)等苂光响应机理进行探针 结构的合理设计. 本文从有机小分子苂光探针的设计、 结构与功能等方面总结和评述了近几年来发展的重要 的检测 RSMs 的线粒体荧光探针及其生物学应用.

\section{1 单功能线粒体定位探针}

早期发展的单功能线粒体探针仅可以对细胞中的 线粒体进行定位和荧光成像, 通过观察其形态变化以判 断细胞的调亡等过程. 线粒体探针在设计时通常引入经 典的亲脂阳离子定位基团, 如 TPP 和季铵盐, 使得探针 能够穿过磷脂双分子层并于跨膜电势驱动下在线粒体 基质中积累. 探针分子中阳离子的存在还有利于增强其 水溶性以透过细胞膜进入细胞内部, 实现线粒体成 像 ${ }^{[11]}$.

近年来，唐本忠等 ${ }^{[2]}$ 基于聚集诱导发光(AIE)机理 设计合成了探针 1 对线粒体进行长时间示踪和成像. 该 探针克服传统商业用线粒体示踪探针光稳定性差、价格 昂贵、制备复杂等不足. 研究结果显示, 其固态在纳米 尺度聚集, 发射强蓝光, 颗粒聚集过程恰好与细胞定位 条件相一致, 这使得探针 $\mathbf{1}$ 易于穿过细胞膜在线粒体聚 集, 实现了活体宫颈癌 HeLa 细胞系线粒体成像. 该研

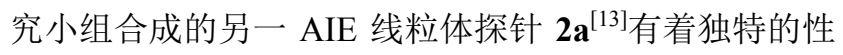
质, 在 $95 \%$ 的水溶液中, 探针 $\mathbf{2 a}$ 的苂光强度和颜色随时 间延长(30 min 内)而发生变化. 通过透射电子显微镜 (TEM) 和电子折射仪(ED)进行观察分析发现, 溶液中探 针 2a 可能为了适应晶格进行构象扭转从而结晶, 由不 定形态转变为层状晶体, 荧光发生蓝移, 且强度增强. 最近，他们基于 AIE 机理合成的线粒体苂光探针 $\mathbf{2 b}^{[14]}$ 能够发出红色苂光, 并可通过苂光信号的不同反映膜电 势的变化.
Neto 等 ${ }^{[3]}$ 合成的中性分子的线粒体苂光探针 3 比商 业用品 MitoTracker Red 对线粒体的选择性定位更佳且 荧光更强、光稳定性更好, 实现了乳腺癌 MCF-7 细胞线 粒体的示踪成像. 探针分子结构中苯并噻二唑基团可与 线粒体内膜上的一类转运蛋白腺嘌呤核苷酸转位酶相 结合, 推测有可能是为该探针对线粒体有高选择性的原 因.

彭孝军等 ${ }^{[15]}$ 设计合成的探针 $\mathbf{4 a}$ 具有细胞毒性低、 光稳定性高、对 $\mathrm{pH}$ 敏感度低等优点, 实现了 $\mathrm{HeLa} /$ MCF-7 细胞线粒体的定位. 值得注意的是该定位过程 与线粒体膜电势无关，探针分子是通过被线粒体膜吞噬 至线粒体内部从而实现成像. 在探针 $\mathbf{4 a}$ 的基础上该课 题组 ${ }^{[16]}$ 在 BODIPY 上引入噻吩基团合成得到了探针 $\mathbf{4 b}$, 该探针水溶性增强且发射波长红移至近红外区域，可用 于观察 HeLa/MCF-7 细胞线粒体形态的变化.

在检测组织线粒体方面, 双光子苂光探针的优点十 分显著, 这种成像利用双光子显微镜(TPM)进行, 该技 术可使激发光穿透更深以到达目标细胞器线粒体. 这就 要求探针的双光子吸收截面 $\left(\delta_{\mathrm{TPA}}\right)$ 大, 从而降低对激光 功率的要求. 其中咔唑由于具有刚性平面和长的共轭体 系成为双光子吸收应用中的优良苂光团. Mak 等 ${ }^{[17]}$ 以咔 唑为荧光团设计合成了三种在水溶液中 $\delta_{\mathrm{TPA}}$ 大、亮度和 对比度高的荧光探针 $5 a \sim 5 c$, 其中 $5 a 、 5 b$ 可定位于线 粒体, $\mathbf{5 c}$ 可选择性定位于溶酶体. 于晓强等 ${ }^{[18]}$ 基于给体$\pi$ 共轭-受体(D- $\pi-A)$ 原理设计合成的双光子线粒体荧光 探针 6 细胞毒性小、能够精确定位于线粒体. 同样地, 探 针 $\mathbf{6}$ 也以咔唑为苂光团, 在有机溶剂中 $\delta_{\mathrm{TPA}}$ 较大, 探针 性能优良.

为了克服 $\mathrm{D}-\pi-\mathrm{A}$ 型探针中普遍存在的光稳定性差 等缺陷, $\mathrm{Kim}$ 等 ${ }^{[19]}$ 设计了探针 7, 将容易发生顺反异构 的碳碳双键固定在碳环中从而提高了其光稳定性. 同 时，分子结构上线性环状共轭体系的增长使得 ICT 效应 增强, 双光子荧光辐射红移至红光区域. 实验证明该探 针可用于大脑皮质神经元和鼠的海马区组织中线粒体 的三维成像.

另外, 单功能线粒体荧光探针在疾病治疗方面也表 现出优异的性能. Cheng 等 ${ }^{[20]}$ 合成的线粒体苂光探针 $\mathbf{8}$ 能够将荧光成像、癌症治疗融为一体. 该探针同时含有 两个线粒体定位基团(TPP 和吡啶季铵盐), 能够更精确 地定位于各种癌细胞线粒体进行成像诊断, 并诱发癌细 胞调亡坏死、抑制其增殖. 探针 $\mathbf{8}$ 中电负性氟原子的引 入使得定位成像和杀伤癌细胞的能力均有显著提高.

在线粒体富集的心肌细胞中, 病态线粒体自噬是心 肌保护的重要过程. Josephson 等 ${ }^{[21]}$ 合成了一系列 TPP 荧 


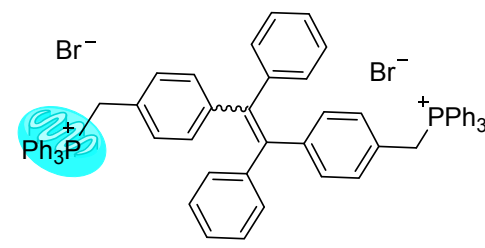

1 TPE-TPP

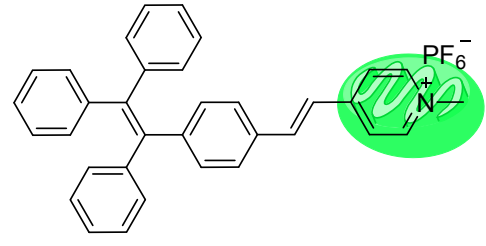

2a TPE-Py

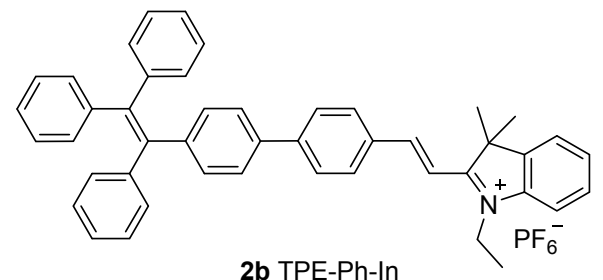

2b TPE-Ph-In
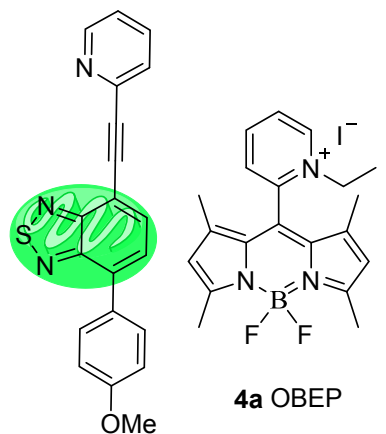

4a OBEP

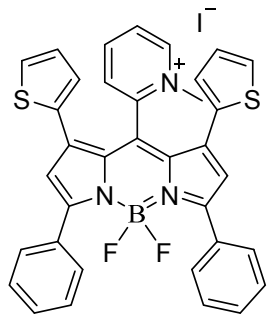

4b M-DPT

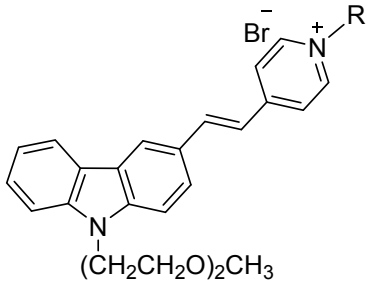

5a SPBN: $\mathrm{R}=\left(\mathrm{CH}_{2}\right)_{4} \stackrel{+}{\mathrm{N}}\left(\mathrm{C}_{2} \mathrm{H}_{5}\right)_{3} \mathrm{Br}^{-}$ 5b SPHP: $\mathrm{R}=\left(\mathrm{CH}_{2}\right)_{6} \stackrel{+}{\mathrm{PPh}} \mathrm{B}_{3} \mathrm{Br}$

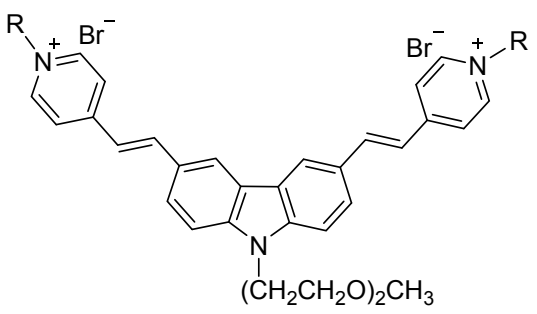

5c VPBN: $\mathrm{R}=\left(\mathrm{CH}_{2}\right)_{4} \stackrel{+}{\mathrm{N}}\left(\mathrm{C}_{2} \mathrm{H}_{5}\right)_{3} \mathrm{Br}$

3 Splendor

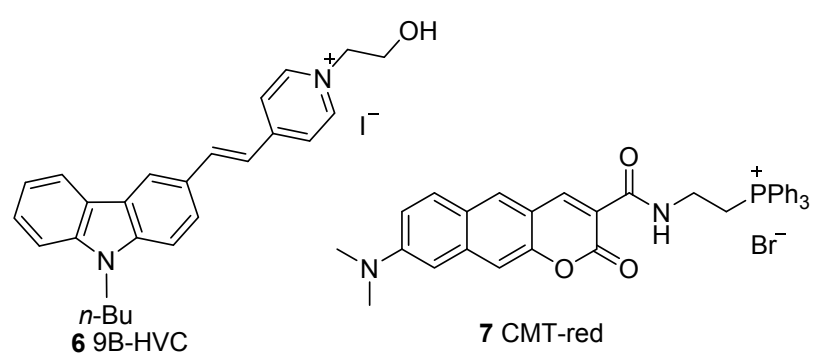<smiles>Fc1ccc2[nH]cc(CCc3cc[n+](CCCCPc4ccccc4)cc3)c2c1</smiles>

8 FF16-TPP<smiles></smiles>

9a TPP-green

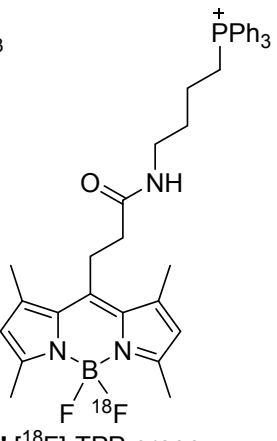

9a' $\left.{ }^{18} \mathrm{~F}\right]-\mathrm{TPP}$-green<smiles></smiles>

9b TPP-Red<smiles>CN1/C(=C/C=C/C=C/C2=[N+](CCCCC(=O)NCCCC[PbH])c3ccc4ccccc4c3C2(C)C)C(C)(C)c2c1ccc1ccccc21</smiles>

图 1 单功能线粒体荧光探针结构

Figure 1 Structures of several mono-functionalized fluorescent probes targeted on mitochondria

光染料 $9 a \sim 9 c$, 研究表明这三种 TPP 探针并不依赖线粒 体膜电势定位, 其定位是由于进入细胞膜后与线粒体中 表达的一种目前未知的底物相结合而引起的, 这使得该 探针有望用于示踪线粒体自噬过程并对其进行成像. 其 中 9c 为近红外苂光探针, 其荧光发射区域与内源荧光 物质(如血红蛋白、色氨酸、NADH 等)不同从而降低了 生物背景荧光的影响. 探针 $9 \mathrm{a} \sim 9 \mathrm{c}$ 如多数亲脂阳离子 探针一样可被功能正常的心肌细胞吸收, 而不能被缺血
的心肌所吸收. 通过进一步注射放射性 ${ }^{18} \mathrm{~F}$ 标记的探针 $9 \mathbf{a}^{\prime}$ 和使用 PET/CT 仪, 活鼠心脏中心肌缺血的部位能够 被有效辨识, 并可据此进一步分析心肌梗塞部位.

Xing 等 ${ }^{[22]}$ 使用菁染料 $(\mathrm{Cy} 3 / \mathrm{Cy} 5)$ 通过点击化学成功 地标记了唾液酸单糖(10a)和唾液酸三糖(10b)(图 2). 细 胞成像实验表明 10a 定位于 PC-12 细胞的线粒体, 是一 种水溶性良好的线粒体探针. $10 \mathrm{~b}$ 整体带有两个负电荷, 很难进入细胞内部. 它可以与细胞膜表面涎免凝素 


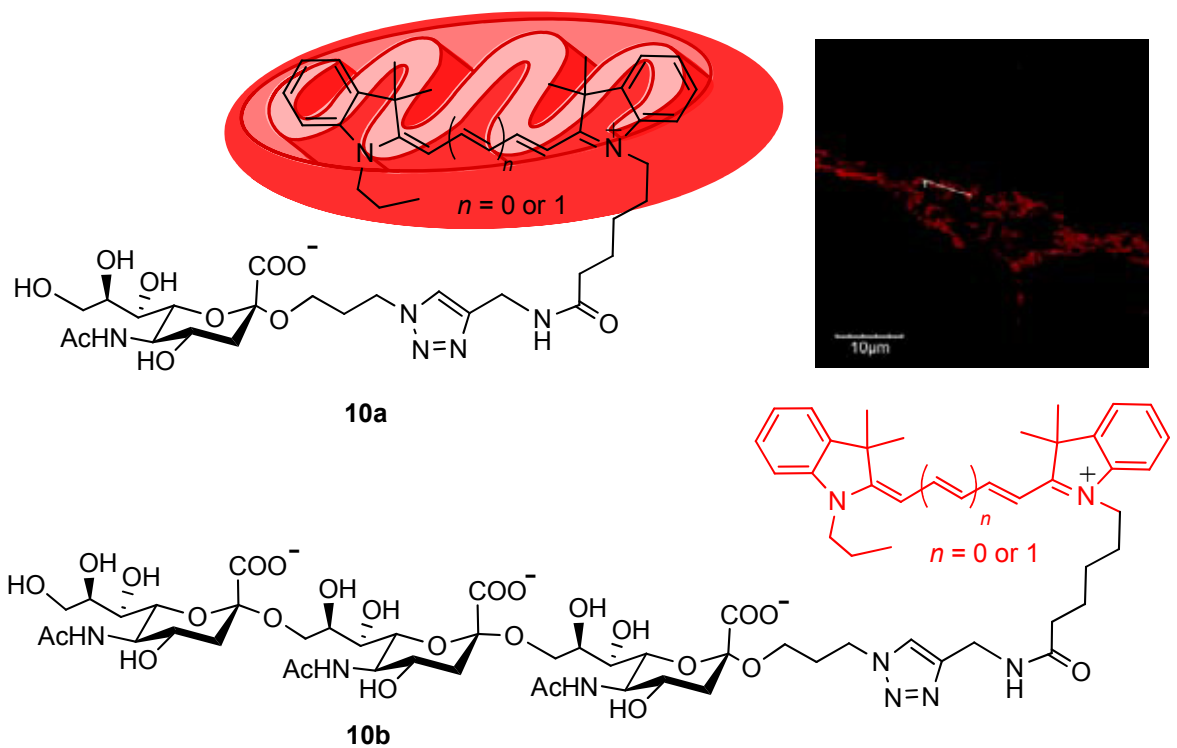

图 2 菁染料标记的线粒体和细胞膜苂光探针结构图

Figure 2 The structures of cyanine labeled mitochondria- and membrane targetable fluorescent Probes

(siglec)相结合, 是一种细胞膜荧光探针. 以 $10 b$ 为成像 工具, 通过 $\mathrm{Cy} 3$ 和 Cy5 之间的 FRET 效应, 获得了涎免 凝素在细胞膜上的空间分布图像. 该研究结果表明, 以 菁染料作为线粒体定位基团, 探针分子中唾液酸糖基单 元的数目对探针的最终性能具有重要的影响.

\section{2 检测活性氧物种的线粒体荧光探针}

在单功能线粒体探针的研究基础上, 近年来, 既能 定位于线粒体又能检测线粒体中各类 RSMs 的双功能荧 光探针也层出不穷. 线粒体在通过电子传递链为细胞提 供能量的同时, 也产生了反应副产物一活性氧物种 ROS. ROS 是一把双刃剑一一它可以作为信号载体参与 线粒体内的生物氧化还原反应、维持免疫应答; 但是, 如果线粒体内电子传递链产生过量的 ROS, 则会造成 氧化应激从而破坏细胞中包括脂质、蛋白质和核酸在内 的各种物质, 使细胞正常的生理过程受到影响, 导致生 物体衰老或疾病. 因此, 线粒体内 ROS 的浓度和时空 分布能够影响细胞乃至生物体的诸多生理过程, 相关研 究对深入揭示线粒体生命活动规律起着重要的作用, 越 来越受到研究者们的关注.

ROS 在生物体内存在时间短、浓度低, 常规检测方 法不易实现，设计并合成定位于线粒体的荧光探针，并 实现对 ROS 的实时监测与成像成为生物探针研究领域 的研究热点.

\section{1 检测高活性氧物种的线粒体荧光探针}

线粒体内高活性氧物种 $\left(\mathrm{hROS}\right.$, 如: $\mathrm{ClO}^{-}, \mathrm{ONOO}^{-}$,
- $\mathrm{OH})$ 能够直接氧化核酸、蛋白质和脂质，在活体细胞中 有着特殊的生物功能, 其参与的详细生理过程仍有待深 入研究.

为满足检测线粒体内各种痕量 $h R O S$ 的要求，彭孝 军等 ${ }^{[23]}$ 报道了高选择性检测 $\mathrm{hROS}\left(\mathrm{ClO}^{-}, \cdot \mathrm{OH}\right)$, 抗其他 ROS 干扰的荧光探针 11a. 该探针以苯并噻嗪环为受体, 以环上电子密度大的硫原子和与半菁相连的双键为反 应位点, 被 $\mathrm{hROS}$ 氧化后伴随着半菁的 $\pi$ 共轭体系被破 坏，使得探针对水溶液中 hROS 产生高选择性的比率型 苂光响应，从而能够对 HeLa 细胞线粒体内源 hROS 进 行成像. 检测特定的 hROS $\left(\mathrm{ClO}^{-}\right)$的探针(图 3)的出现 使得对特定分子的生理过程研究成为可能. 同样基于苯 并噻嗪, 最近尹桂等 ${ }^{[2]}$ 设计合成了水溶性、生物兼容性 好的探针 $11 \mathrm{~b}$, 能够选择性检测线粒体内源性 $\mathrm{HClO} / \mathrm{ClO}^{-}$.

彭孝军等 ${ }^{[25]}$ 报道了基于肜基为反应基团的关开型 探针 12, 加入 $\mathrm{NaClO}$ 前探针溶液呈粉色、几乎无苂光; 与 $\mathrm{NaClO}$ 快速反应后蓝移至黄色, 荧光强度增强 35 倍. 该探针将经典的 TPP 线粒体定位基引入 BODIPY 的 meso 位, 实现了活体细胞 MCF-7 线粒体内快速、高灵 敏度和高选择性 $\mathrm{ClO}^{-}$监测.

随后，彭孝军等 ${ }^{[26]}$ 又设计合成了以 $\mathrm{Cy} 7$ 为苂光团的 近红外苂光探针 13, 该探针结构中 $\mathrm{Se}$ 未参与苂光团共 轭体系，仅作为氧化还原反应位点以实现 $\mathrm{ClO}^{-}$的高选 择性检测. 经实验验证，探针 13 可用于胎牛血清中 $\mathrm{HClO}$ 的高选择性检测, 并可用于活体大鼠 $\mathrm{HClO}$ 的分 子成像. 


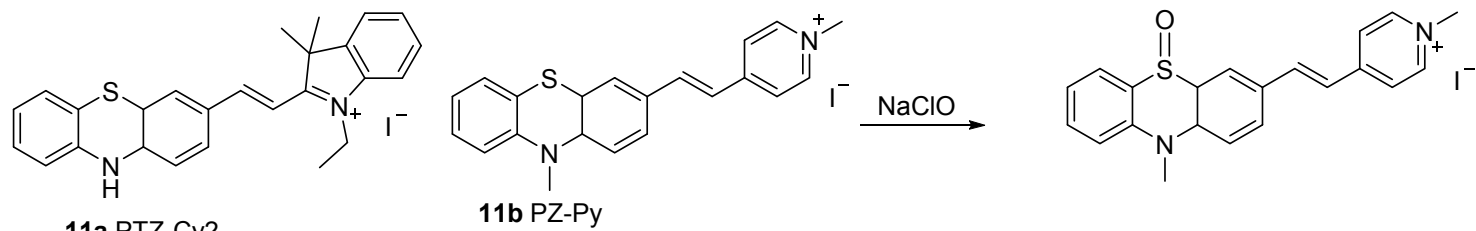

11a PTZ-Cy2<smiles></smiles><smiles></smiles><smiles></smiles>

12 MitoClO<smiles>CCN1/C(=C\C=C2/CCCC(/C=C/C3=[N+](CC)c4ccccc4C3(C)C)=C2N2CC[Se]CC2)C(C)(C)c2ccccc21</smiles><smiles></smiles>

图 3 检测 $\mathrm{hROS}$ 的线粒体苂光探针结构与响应原理

Figure 3 Targetable fluorescent probes in the mitochondria for detection of hROS: structures and responsing reactions

李坤等 ${ }^{[27]}$ 设计合成了检测 $\mathrm{ClO}^{-}$和活体成像的关开 型探针 14. 探针结构中作为定位基团的吡啶盐部分通 过柔性链与罗丹明相连接, 从而避免吡啶盐常见的易与 线粒体中负电性物种发生静电作用而影响苂光性能的 缺点. 探针与 $\mathrm{ClO}^{-}$反应后酰肼部位发生氧化水解, 从而 使得探针的荧光恢复. 研究结果显示探针 14 可用于 $\mathrm{HeLa}$ 细胞线粒体内 $\mathrm{ClO}^{-}$的分子成像, 并能进一步用于 裸鼠活体成像.

\section{2 检测过氧化氢的线粒体荧光探针}

在诸多 $\mathrm{ROS}$ 中, $\mathrm{H}_{2} \mathrm{O}_{2}$ 是细胞中重要的 $\mathrm{ROS}$, 细胞线 粒体中 $\mathrm{H}_{2} \mathrm{O}_{2}$ 过量容易导致多种疾病发生，对细胞内 $\mathrm{H}_{2} \mathrm{O}_{2}$ 的含量和分布进行监测与成像有利于疾病的前期 篮查与诊断. 一般而言, 喼酸频哪醇酯常被选为选择性 检测 $\mathrm{H}_{2} \mathrm{O}_{2}$ 的反应位点, $\mathrm{H}_{2} \mathrm{O}_{2}$ 与硼酸酯反应后导致探针 分子结构的变化，从而引发荧光响应(图 4).

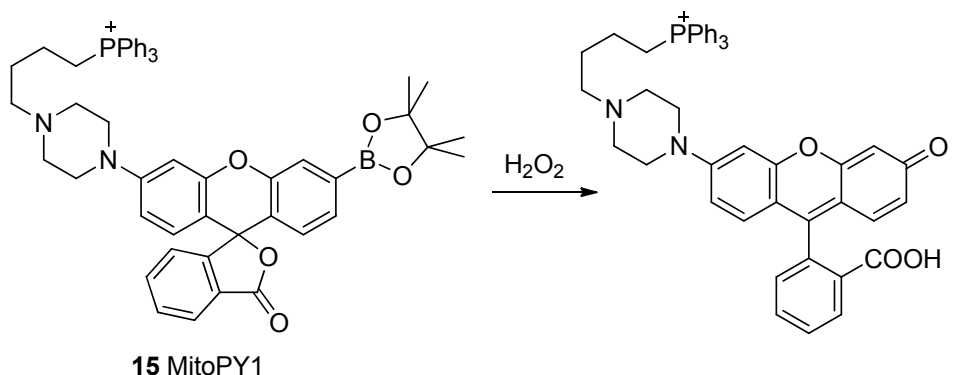<smiles>CNc1ccc2cc(-c3nc4ccc(C(=O)NCC[PH2+])cc4s3)ccc2c1</smiles>

图 4 检测 $\mathrm{H}_{2} \mathrm{O}_{2}$ 的线粒体苂光探针结构与响应原理

Figure 4 Targetable fluorescent probes in the mitochondria for detection of $\mathrm{H}_{2} \mathrm{O}_{2}$ : structures and responsing reactions 
Chang 等 ${ }^{[28,29]}$ 报道的探针 $\mathbf{1 5}$ 能高选择性地定位于 哺乳动物细胞系的线粒体上, $\mathrm{H}_{2} \mathrm{O}_{2}$ 与探针反应后将其变 为酚, 继而诱发了环内酯的开环反应导致探针分子的 荧光团共轭体系增强, 发射较强苂光. 探针 15 可用于 $\mathrm{HeLa}$ 细胞中 $\mathrm{H}_{2} \mathrm{O}_{2}$ 的实时成像.

在此基础上, Cho 等 ${ }^{\left[{ }^{30]}\right.}$ 也以苯硼酸频哪醇酯为识别 基团, 通过 $\mathrm{H}_{2} \mathrm{O}_{2}$ 氧化引发探针分子分解产生荧光信号 红移从而实现对其高选择性检测. 实验研究显示, 他们 设计合成的定位于线粒体检测 $\mathrm{H}_{2} \mathrm{O}_{2}$ 的双光子比率型苂 光探针 16 可以对大鼠海马区切片中的 $\mathrm{H}_{2} \mathrm{O}_{2}$ 进行成像, 克服了探针 15 不能深入组织成像的缺点, 突显出 16 作 为双光子荧光探针在应用上的优势.

同样以苯硼酸频哪醇酯为识别基团, 邵士俊等 ${ }^{[1]}$ 设计合成了关开型检测 $\mathrm{H}_{2} \mathrm{O}_{2}$ 的探针 17, 该探针以 $\mathrm{C}-\mathrm{N}$ 键作为连接臂, 具有水溶性和生物兼容性高等优点, 可 用于活体内分子成像. 由于探针 17 分子中存在 PET 效 应, 未与 $\mathrm{H}_{2} \mathrm{O}_{2}$ 作用前探针的苂光量子产率很低; 与 $\mathrm{H}_{2} \mathrm{O}_{2}$ 反应后, 吡啶取代 BODIPY 部分荧光恢复, 荧光强 度增强 58 倍. 探针 $\mathbf{1 7}$ 可用于肝癌细胞 HepG2、正常肝 细胞 $\mathrm{LO} 2$ 和活体斑马鱼的 $\mathrm{H}_{2} \mathrm{O}_{2}$ 检测与成像, 并能对 $\mathrm{HeLa}$ 细胞中探针附着位置的 $\mathrm{H}_{2} \mathrm{O}_{2}$ 流量作出 “turn-on” 响应, $\mathrm{H}_{2} \mathrm{O}_{2}$ 含量越高, 荧光强度越大.

\section{3 检测一氧化氮的线粒体荧光探针}

$\mathrm{NO}$ 是心血管、神经、免疫系统细胞中一种常见的 信使, 而线粒体是合成细胞内源 NO 的主要场所, 这就 使得线粒体 $\mathrm{NO}$ 的实时监测十分重要.

肖义等 ${ }^{[2]}$ 报道了首个可以定位于线粒体的高选择 性检测 NO 的关开型苂光探针 $\mathbf{1 8}$ (Eq. 1). NO 氧化并打 开罗丹明的内酰胺螺环使得探针荧光增强 60 倍, 该探 针可用于人乳腺癌 MCF-7 细胞线粒体内源 NO 的成像.

郭炜等 ${ }^{[33]}$ 设计合成了结构简单的关开型探针 19 , 将邻苯二胺引入了荧光团派洛宁上作为 $\mathrm{NO}$ 的受体. 由 于反应产物三唑上不存在多余的氢原子, 排除了 $\mathrm{pH}$ 和 其余干扰物质(如 DHA 等)对 NO 苂光响应的干扰. 值得 注意的是, 借助还原性物种 Cys 和 GSH, 发红色苂光的 探针氧化产物可以进一步反应分别生成绿色和红色荧 光产物. 使用探针 19 实现了双通道选择性检测小鼠黑
色素瘤细胞 B16 及小鼠单核巨噬细胞白血病细胞 RAW264.7 中线粒体内源及外源 NO 并进行成像(图 5).

\section{4 检测超氧阴离子自由基的线粒体荧光探针}

线粒体呼吸过程中, 电子传递链上的电子传给氧分 子则产生 $\mathrm{O}_{2}^{--}$. 作为线粒体中首先合成的 $\mathrm{ROS}, \mathrm{O}_{2}^{--}$会 迅速转变为 $\mathrm{H}_{2} \mathrm{O}_{2}$, 这就意味着超氧负离子的浓度反映 着 $\operatorname{ROS}$ 整体含量水平. 因此, 设计合成高效的超氧阴离 子苂光探针也十分重要. 唐波等 ${ }^{[34]}$ 报道了检测超氧阴 离子的双光子探针 20 (Eq. 2). 该探针以苯并噻唑啉为 超氧阴离子的高选择性受体, 被超氧阴离子氧化后共轭 体系增大, 苂光增强; 以荡作为双光子苂光团, 能被双 光子激发用于 $\mathrm{HepG} 2 / \mathrm{HeLa}$ 细胞和活体大鼠的高效高选 择性超氧负离子的分子成像.

\section{3 检测还原性物种的线粒体荧光探针}

正常状态下, 线粒体中的抗氧化系统可以有效地清 除过量的 ROS, 使 ROS 和还原性物种(如谷胱甘肽 GSH 和硫化氢 $\mathrm{H}_{2} \mathrm{~S}$ 等含巯基的化合物)达到一种精确的平衡. 为监测线粒体内 $\mathrm{ROS}$ 和还原性物种的平衡, 针对还原 性物种检测的线粒体荧光探针也层出不穷.

\section{1 检测巯基化合物的线粒体荧光探针}

其中，作为线粒体还原系统中最常见的还原性物 种, GSH 等颈基化合物的检测成为研究热点(图 6). 唐波 等 ${ }^{[35]}$ 合成出了一种近红外苂光探针 $\mathbf{2 1}$, 该探针能可逆、 高效、高选择性地检测一对具有代表性的氧化还原 对一- $\mathrm{H}_{2} \mathrm{O}_{2}$ 和 $\mathrm{GSH}$. 该探针以 $\mathrm{Se}-\mathrm{N}$ 键为检测位点, 随着 GSH 的加入, 探针 21 的苂光淬灭; 再加入适量 $\mathrm{H}_{2} \mathrm{O}_{2}$, 苂光恢复. 研究表明若细胞 $\mathrm{HepG} 2$ 线粒体中 $\mathrm{H}_{2} \mathrm{O}_{2}$ 含量过高、 $\mathrm{GSH}$ 含量较低, 细胞将面临死亡. 探针 21 可以对 HepG2/HL7702 细胞和斑马鱼幼体内的氧化 还原态进行实时成像.

Kim 等 ${ }^{[36]}$ 对线粒体中 GSH 的不可逆检测进行了报 道, 其研究组合成了双光子比率型苂光探针 22. GSH 的 亲核进攻使得二硫键异裂，进而引发探针的酰胺部分异 裂，探针的荧光发射红移而实现响应. 探针 22 可以对 HeLa 细胞系和大鼠海马区脑片线粒体中的 GSH 成像.

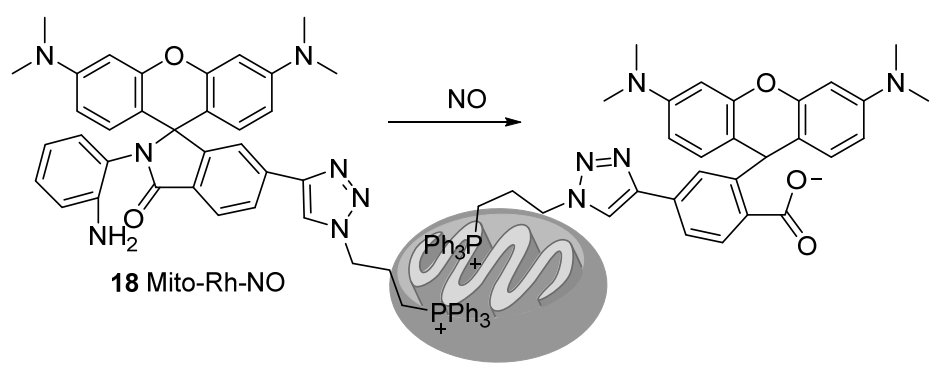



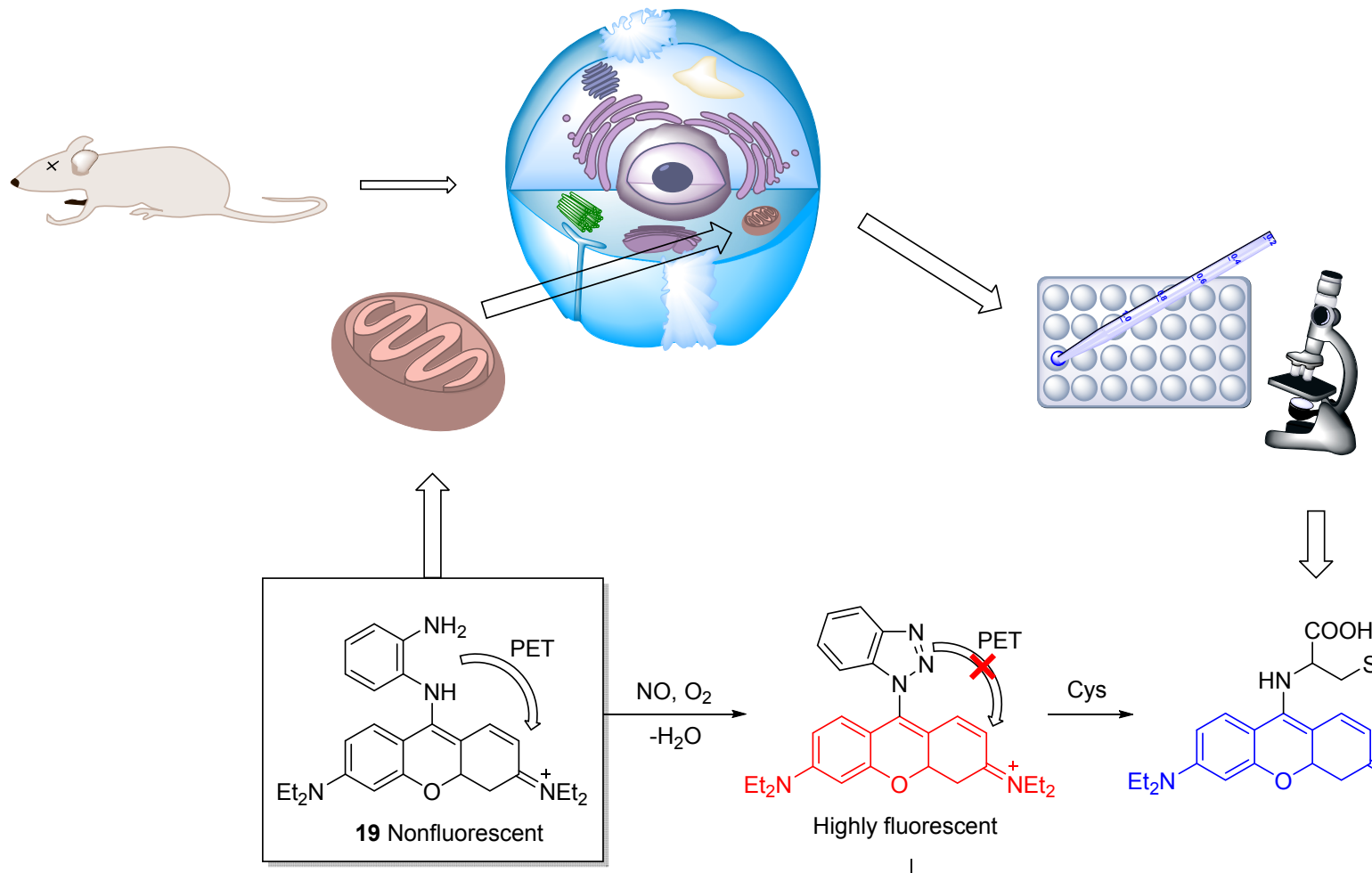<smiles>C=[N+](CC)C1=CC=C2C(SCC(NC(=O)CCC(N)C(=O)O)C(=O)NCC(=O)O)=Cc3ccc(N(CC)CC)cc3OC2C1</smiles>

图 5 双通道选择性检测线粒体内源 $\mathrm{NO}$ 的原理

Figure 5 Targetable fluorescent probes in the mitochondria for detection of NO generating from mitochondria: structures and responsing reactions

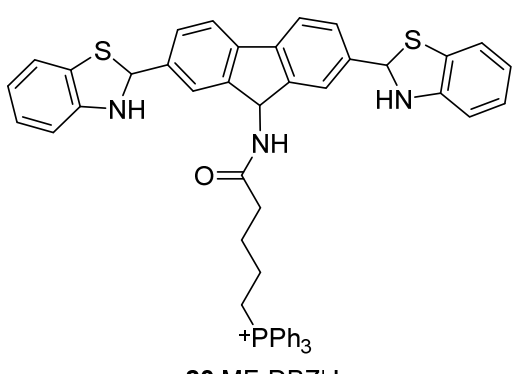

20 MF-DBZH

为了达到在生物样品中进行无创成像的目标，Kim 等 ${ }^{[37]}$ 还设计合成了检测 GSH 的近红外荧光探针 $\mathbf{2 3}$, 并 对探针的高选择性检测进行了探讨. 探针 23 中 $\mathrm{Cy} 7$ 与 偶氮部分由于 PET 过程使其苂光发射受阻, 因此偶氮基 团不仅是 GSH 的选择性反应位点, 还是荧光淬灭基团. 只有 GSH 与探针 23 反应才能有效解除 PET 禁阻, 使得 探针荧光恢复; 而半胱氨酸和同型半胱氨酸不能使探针 的荧光恢复. 探针 $\mathbf{2 3}$ 可用于活体 HeLa 细胞线粒体成像.<smiles>O=C(CCCC[PH+](c1ccccc1)c1ccccc1)NC1c2cc(-c3nc4ccccc4s3)ccc2-c2ccc(-c3nc4ccccc4s3)cc21</smiles>

基于半胱氨酸(Cys)诱导的自然化学连接(NCL)-串 联成环反应和静电相互作用，郭炜等 ${ }^{[38]}$ 设计了可抗同 型半胱氨酸(Hcy)和 GSH 干扰, 能高选择性检测 Cys 的 比率型线粒体苂光探针 24a. 该探针与 Cys 经过 NCL串联成环反应后, 苂光蓝移, 在 644 和 $494 \mathrm{~nm}$ 处出现比 率型变化. 实验研究表明探针 24a 可用于人肝癌细胞

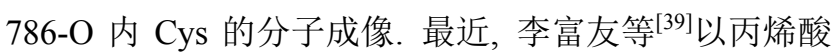
酯作为 Cys 识别基团和荧光淬灭基团的近红外线粒体探 


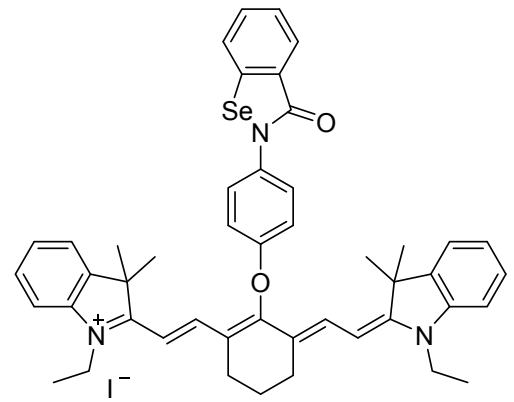

21 Cy-O-Eb<smiles>CCOCCOCCOC(=O)N(C)c1ccc2cc(-c3nc4ccc(C(=O)NCCc5ccccc5)cc4s3)ccc2c1</smiles>

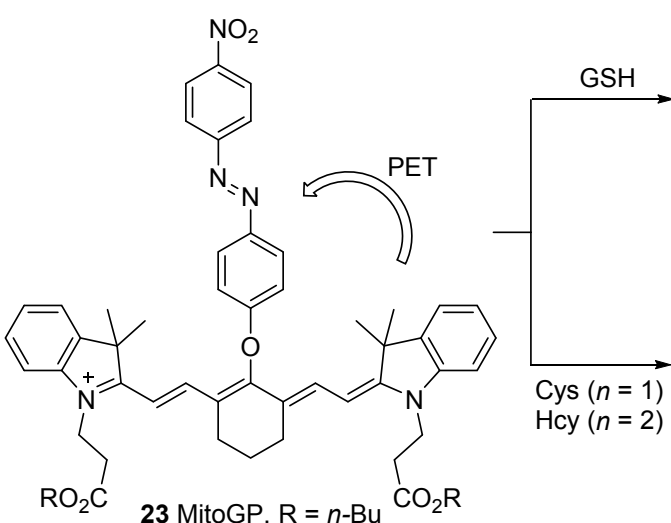

None fluorescence

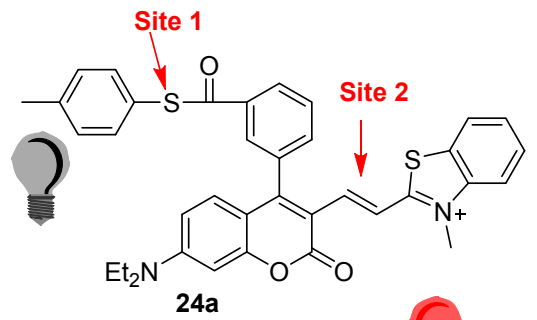

Cys<smiles></smiles><smiles>C=CC(=O)OC1CCC2(CC2)C1</smiles>

$24 b$

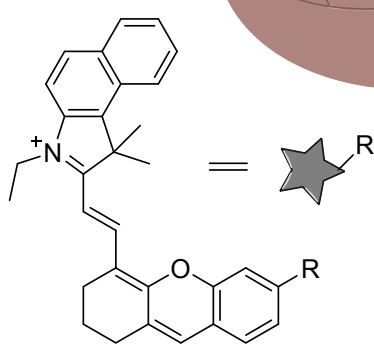

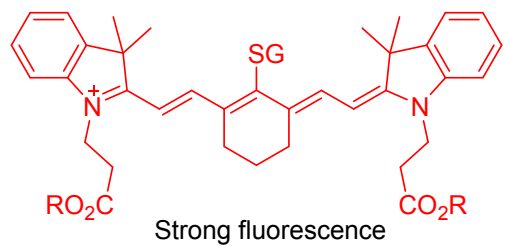<smiles>CC(C)=O</smiles>

$\stackrel{\mathrm{GSH}}{\longrightarrow}$

$\mathrm{Et}_{2} \mathrm{~N}$

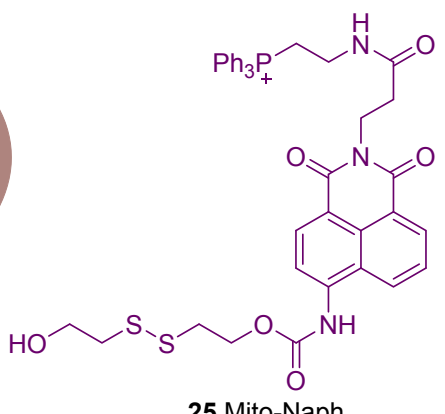

25 Mito-Naph<smiles>CCN1/C(=C/C=C2\CCCC(/C=C/C3N(CC)c4ccccc4C3(C)C)=C2Oc2ccc(NCO)cc2)C(C)(C)c2ccccc21</smiles>

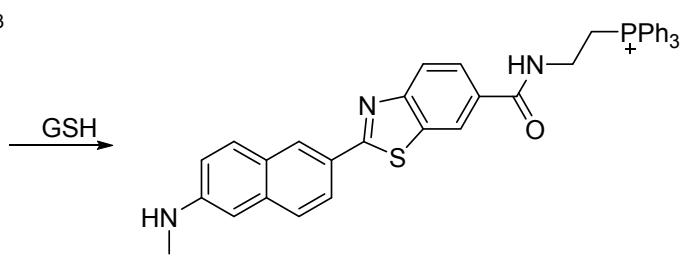

图 6 检测 GSH 等颈基化合物的线粒体荧光探针结构与响应原理

Figure 6 Targetable fluorescent probes in the mitochondria for detection of sulfhydryl compounds (GSH etc.): structures and responsing reactions 
针 24b. 该探针能够高选择性响应线粒体内源性 Cys, 苂光信号显著增强. 24b 能够评价活细胞线粒体内的氧 化应激，具有潜在的生物应用前景.

细胞中和细胞间的硫氧还蛋白 Trx 过量可能导致癌 症、心血管疾病等病症. Kang 等 ${ }^{[40]}$ 设计并合成了能够检 测线粒体中大分子硫醇 $\operatorname{Trx}$ 的探针 25. 其中二硫键为硫 醇的检测位点, 能够高选择性检测 Trx. 探针与 Trx 快速 反应后, 菼光由蓝色(472 nm)红移至绿色(540 nm), 苂 光强度增强 14 倍, 可用于 HepG2 细胞线粒体成像.

\section{2 检测硫化氢的线粒体荧光探针}

还原性物种中除硫醇外, $\mathrm{H}_{2} \mathrm{~S}$ 在线粒体氧化应激中 保护细胞免于紊乱和死亡. 检测线粒体 $\mathrm{H}_{2} \mathrm{~S}$ 的探针成为 包括药物治疗在内很多领域的研究热点 ${ }^{[4]}$, 这类探针常 设计为比率型苂光探针(图 7). 何卫江等 ${ }^{[41]}$ 报道了基于 ICT 原理的比率型荧光探针 26. 在中性环境下, 半菁被 $\mathrm{HS}^{-}$亲核加成后共轭体系发生改变, 荧光团由原本的香 豆素、半菁杂合荧光团变为单荧光团香豆素, 苂光发射 由原本的红色 $652 \mathrm{~nm}$ 蓝移至绿色 $510 \mathrm{~nm}$, 达到比率型 检测的目的. 探针 26 不仅可以高选择性检测 $\mathrm{H}_{2} \mathrm{~S}$, 还可 应用于 $\mathrm{H}_{2} \mathrm{~S}$ 在活体 $\mathrm{MCF}-7$ 细胞线粒体中的分子成像.

为了深入组织进行成像, Kim 等 ${ }^{[42]}$ 设计合成的双光 子比率型苂光探针 27. 该探针以氨基甲酸酯为连接基 才, 叠氮基为识别基团, 通过 $\mathrm{H}_{2} \mathrm{~S}$ 对探针还原分解而产 生的荧光红移实现检测. 探针可针对帕金森基因敲除的 星状胶质细胞线粒体中的 $\mathrm{H}_{2} \mathrm{~S}$ 进行检测, 对皮质切片中 的 $\mathrm{H}_{2} \mathrm{~S}$ 进行组织内分子成像.

基于线粒体膜电势的探针常用 TPP 等亲脂性阳离 子定位, 此类探针通常具有依赖膜电势乃至改变膜电势 的不足. 像探针 3 - 类的电中性探针有望克服这一缺陷. 宋钦华等 ${ }^{[4]}$ 亦报道了一电中性的双光子比率型荧光探针 28, 并对其在 MCF-7 细胞线粒体上的定位机理作出推 测一一他们认为探针的线粒体靶向是由于该探针中羟 基(和氨基)与线粒体膜形成氢键，从而附着其上. 探针 28 的叠氮基团被 $\mathrm{H}_{2} \mathrm{~S}$ 还原为氨基, 进而引起酰胺键异 裂, 使探针的 ICT 性质发生变化, 苂光发射从蓝色红移 至绿色，实现线粒体中 $\mathrm{H}_{2} \mathrm{~S}$ 的双光子比率检测.

席真等 ${ }^{[5]}$ 报道了基于 FRET 原理抗硫醇干扰的高选 择检测 $\mathrm{H}_{2} \mathrm{~S}$ 的有机小分子苂光探针 29. 该探针结构中香 豆素荧光团作为 FRET 给体而罗丹明作为 FRET 受体. 探针 29 与 $\mathrm{H}_{2} \mathrm{~S}$ 反应后罗丹明螺环打开, 从而可以观测 到 FRET 过程, $I_{675 \mathrm{~nm}} / I_{590 \mathrm{~nm}} 、 I_{525 \mathrm{~nm}} / I_{465 \mathrm{~nm}}$ 增高, 实现了生 理缓冲条件下比率型苂光检测. 苂光共聚焦实验表明探 针 29 可以定位于线粒体, 并且可用于人体胚胎肾脏细 胞 HEK293 线粒体内源及外源 $\mathrm{H}_{2} \mathrm{~S}$ 的检测与成像.

为消除环境影响、提高荧光分析的准确度及实用性,
袁林等 ${ }^{[43]}$ 设计并合成了基于 FRET 原理的双激发线粒体 比率型探针 30. 随着探针与 $\mathrm{H}_{2} \mathrm{~S}$ 的反应, 香豆素苂光团 上的 2,4-二硝基苯基保护基被脱除，使得从香豆素酚氧 负离子给体到罗丹明受体的 FRET 过程得以发生 $\left(\lambda_{\mathrm{ex}}=\right.$ $402 \mathrm{~nm}$ ), 罗丹明在 $\lambda_{\mathrm{em}}=590 \mathrm{~nm}$ 处的苂光发射峰逐渐增 强. 相比之下罗丹明所在 $\lambda_{\mathrm{ex}}=570 \mathrm{~nm}$ 激发所产生的荧 光发射在反应中几乎不变, 可以作为参照信号, 实现高 选择性比率型检测 $\mathrm{H}_{2} \mathrm{~S}$. 探针 30 可用于 MCF-7 细胞线 粒体中 $\mathrm{H}_{2} \mathrm{~S}$ 的检测与成像.

为检测内源 $\mathrm{H}_{2} \mathrm{~S}$ ，唐波等 ${ }^{[44]}$ 设计合成了高选择性的 近红外苂光探针 31. 探针以甲醛基为氧化还原位点, 在 捕捉 $\mathrm{H}_{2} \mathrm{~S}$ 后发生串联的亲核加成、环化反应而引发探针 的分解, 该探针与 $\mathrm{H}_{2} \mathrm{~S}$ 反应后先由绿色菼光快速转变为 中间体荧光淬灭，最后荧光发射红移至近红外区域，使 得探针产生比率型响应信号. 探针能够高效高选择性地 检测人肺癌 $\mathrm{A} 549$ 细胞中内源的 $\mathrm{H}_{2} \mathrm{~S}$ 并用于分子成像.

\section{3 用于研究电子传递链的线粒体苂光探针}

除了以检测 RSMs 为目的外, 利用探针成像研究线 粒体的氧化呼吸作用及电子传递链也有着独特的意义. 复合体 I 处的电子传递链抑制对 ROS 产生起到重要作 用，而 ROS 过剩导致线粒体机能受到破坏. Takeoka 等 ${ }^{[6]}$ 报道了线粒体苂光探针 32 (Eq. 3) 并结合电子顺磁共振 的使用，实现了线粒体膜系统中呼吸链的氧化还原反应 的可视化. 该探针以一氧化氮自由基 TEMPO 为识别位 点, 线粒体中鱼藤酮的加入使得电子转移被禁止; 此时 如果加入的探针 32, 它将通过复合体 I 介导被 NADH 还 原从而导致荧光显著增强. 探针 32 有望成为研究复合 体 I 和呼吸链动态变化的有效工具.

\section{4 检测金属离子的线粒体苂光探针}

锌离子在生物体系中起着重要作用, 它参与了 DNA 的合成、基因转录、酶催化以及免疫应答等过程, 检测锌离子成为金属离子线粒体荧光探针的研究热点 (图 8). 对于以二-2-吡啶甲基胺(DPA)为锌离子受体的荧 光探针，为了提高其抗镉离子干扰性，常在 DPA 附近设 计其他杂原子参与配位以提高探针与锌离子的配位的 稳定性，从而使其与镉离子相区分.

江华等 ${ }^{[45]}$ 报道了基于阳离子诱导 PET 禁阻原理的 比率型苂光探针 33, 该探针以 DPA 和邻近喹啉上的氮 原子共同作为锌离子的识别基团. 探针与锌离子结合后 形成四配位配合物，荧光发射波长发生蓝移，在生理缓 冲条件下加入锌离子后探针苂光发射强度比 $\left(F_{504 \mathrm{~nm}} /\right.$ $\left.F_{550 \mathrm{~nm}}\right)$ 是未加入时的 5 倍, 实现了线粒体中锌离子的量 化检测. 探针 33 能够可视化高选择性检测活体细胞线 粒体中锌离子变化, 并可用以定量检测小鼠肧胎 $\mathrm{NIH}$ 


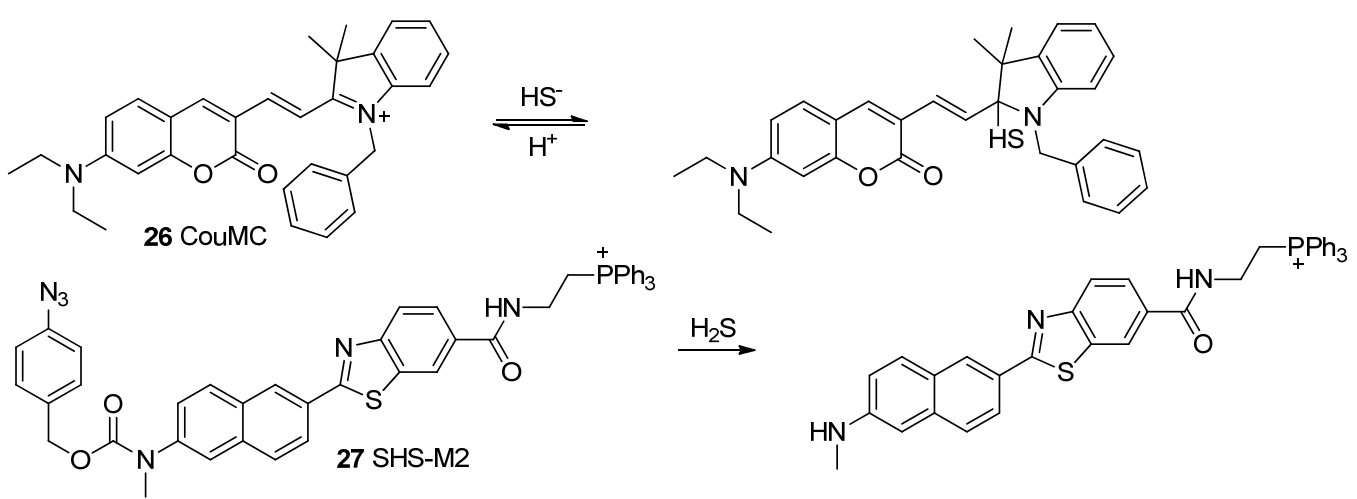

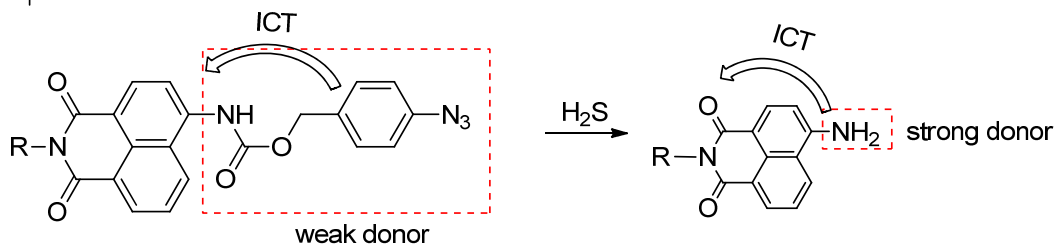

28 AcHS-2: $\mathrm{R}=\mathrm{CH}_{2} \mathrm{CH}_{2} \mathrm{OH}$<smiles></smiles><smiles>C=C=C=CC=CC1=C2C=CC(=[N+](CC)CC)C=C2Oc2cc(N(C)CC)ccc2C1c1ccc(C(=O)N2CCN(C(=O)c3cc4ccc([O-])cc4oc3=O)CC2)cc1</smiles>

30 Ratio- $\mathrm{H}_{2} \mathrm{~S} 1$

$\mathrm{Ab} \&$ Ex only at $570 \mathrm{~nm}$

phenolate form Ab\&Ex at $402 \& 570 \mathrm{~nm}$<smiles>CCN1/C(=C/C=C2\CCCC(/C=C/C3=[N+](CC)c4ccccc4C3(C)C)=C2OC(=O)c2ccccc2C=O)C(C)(C)c2ccccc21</smiles>

31 HS-Cy

highly fluorescent

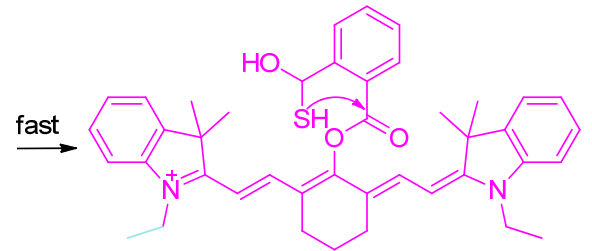

qHS-Cy

fluorescence quenching $\downarrow$ slow<smiles>O=C1SC(O)c2ccccc21</smiles>

ketone-Cy highly fluorescent

图 7 检测 $\mathrm{H}_{2} \mathrm{~S}$ 的线粒体荧光探针结构与响应原理

Figure 7 Targetable fluorescent probes in the mitochondria for detection of $\mathrm{H}_{2} \mathrm{~S}$ : structures and responsing reactions 

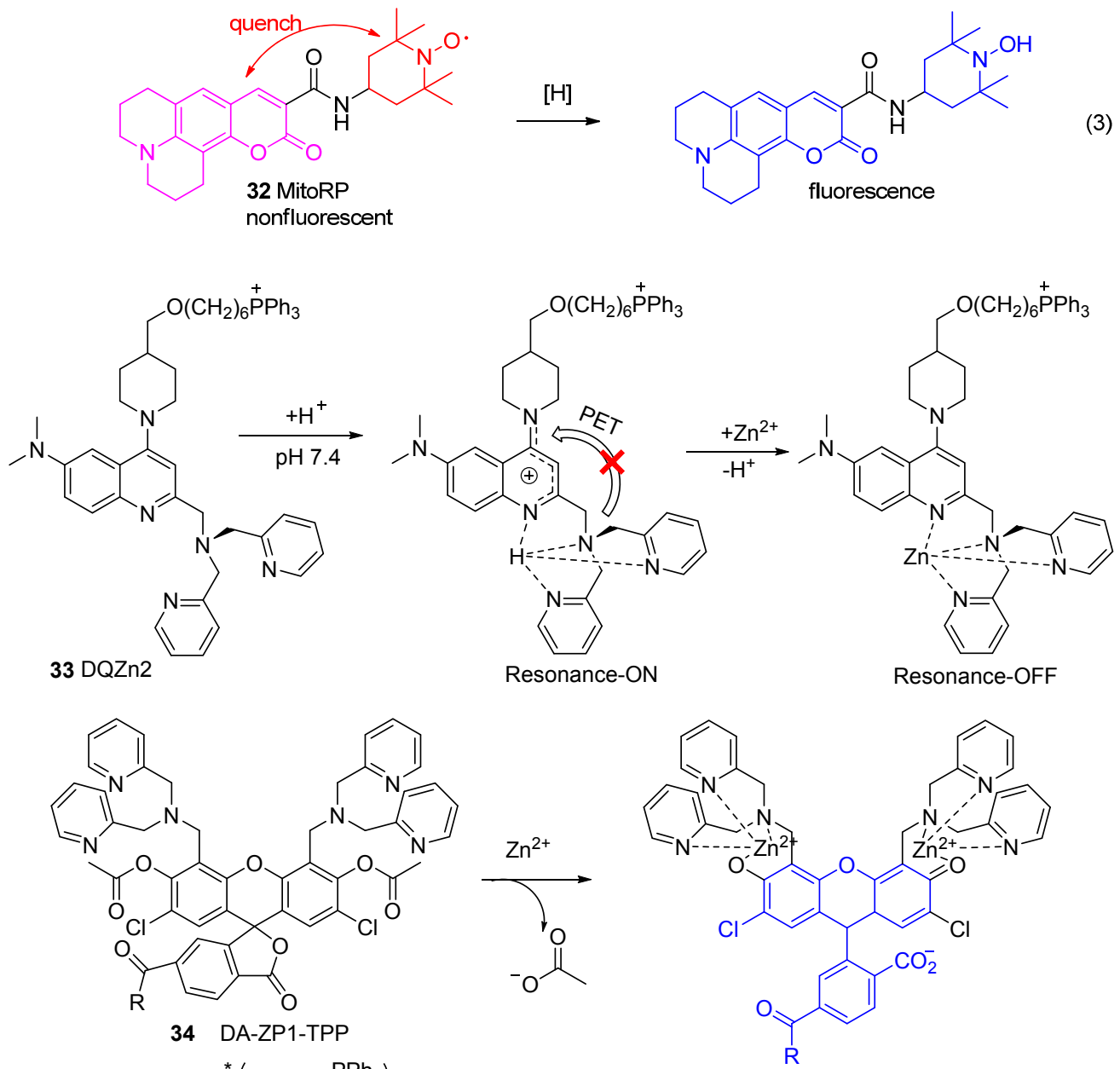

34 DA-ZP1-TPP

$\mathrm{R}={ }^{*}\left(\underset{\mathrm{H}}{\mathrm{N}} \widehat{P}_{+}^{\mathrm{PPh}_{3}}\right)$<smiles>CN(CC(=O)NCCc1ccccc1)c1ccc2cc(-c3nc4ccc(NCCN(Cc5ccccn5)Cc5ccccn5)cc4s3)ccc2c1</smiles><smiles></smiles>

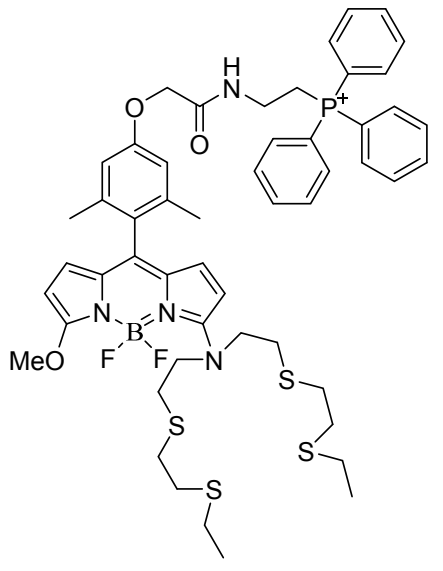

37 Mito-CS1 35 SZn-Mito

36 CR1S

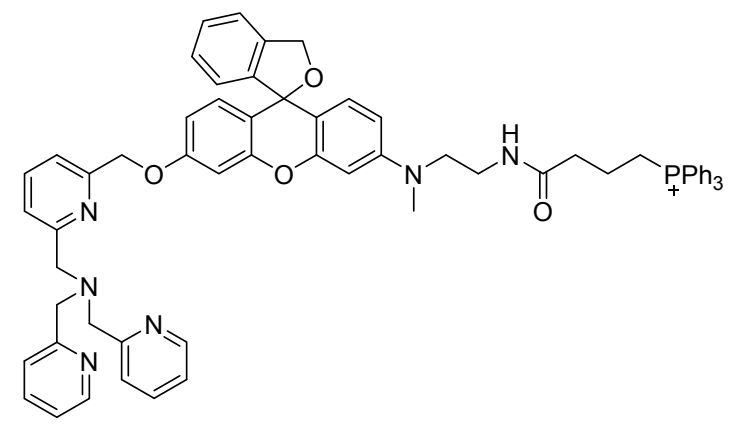

38 RdITPA-TPP

图 8 检测金属离子 $\left(\mathrm{Zn}^{2+}, \mathrm{Hg}^{2+}, \mathrm{Cu}^{+}\right)$的线粒体荧光探针结构与响应原理

Figure 8 Targetable fluorescent probes in the mitochondria for detection of metal ions $\left(\mathrm{Zn}^{2+}, \mathrm{Hg}^{2+}, \mathrm{Cu}^{+}\right)$: structures and responsing reactions 
$3 \mathrm{~T} 3$ 细胞中锌离子浓度.

同样基于 DPA, Lippard 等 ${ }^{[46]}$ 合成了可逆型关开探 针 34, 探针本身由于 PET 效应荧光淬灭, 结合二价锌离 子后形成配合物苂光恢复. 该探针可以在 HeLa 细胞中 成像, 进一步有关该探针应用的研究显示, 与健康的前 列腺细胞不同, 致癌的细胞在线粒体中无法积累游离的 二价锌离子.

更加优良的锌离子检测位点为带有两个亚甲基的 柔性氨基臂的 $N, N^{\prime}$-二-(2-吡啶甲基)乙二胺(DPEA), 这 样的例子有 $\mathrm{Kim}$ 等 ${ }^{[47]}$ 设计的针对 HeLa 细胞系和大鼠的 海马区脑片线粒体中 $\mathrm{Zn}^{2+}$ 检测的关开型双光子苂光探 针 35. 加入 $\mathrm{Zn}^{2+}$ 后该探针的荧光增强 7 倍, 且相应的络 合常数是探针 33、34 的数倍. 值得注意的是, 该探针能 够深入活体组织下 $100 \sim 200 \mu \mathrm{m}$, 且不受其他金属离子 干扰.

采离子作为最危险的重金属污染物之一，易进入人 体, 可穿透进入线粒体, 使人体免疫等功能紊乱. 实现 线粒体内采离子的检测至关重要. 汪鹏飞等 ${ }^{[7]}$ 报道了中 性比率型苂光探针 36. 该探针能够选择性检测线粒体 中的二价采离子, 并成功地实现了 A549 细胞中的 $\mathrm{Hg}^{2+}$ 的分子成像. 探针 36 在加入采离子后发生脱硫反应, 本 身香豆素的蓝色荧光 $430 \mathrm{~nm}$ 逐渐红移至罗丹明的红色 荧光 $653 \mathrm{~nm}$. 发射光谱中高达 $223 \mathrm{~nm}$ 的位移正是由于 探针结构中香豆素和罗丹明稠环结构巧妙设计所致.

由于调节线粒体铜离子的浓度对于维持细胞代谢
十分重要, 实时监测线粒体铜离子动态浓度的苂光探针 可作为研究这种氧化还原活性金属在健康和疾病状态 下时空分布的有效工具. 基于 ICT 机理, Chang 等 ${ }^{[48]}$ 报 道了一个新型的检测 $\mathrm{Cu}^{+}$的关开型线粒体荧光探针 37. 该探针以硫醚基团为识别基团，对水溶液中 $\mathrm{Cu}^{+}$具有高 选择性, 加入 $\mathrm{Cu}^{+}$后探针 37 的苂光强度增强 10 倍. 这 一探针的使用可实现在模式细胞系 HEK 293T 和人体成 纤维细胞中线粒体的 $\mathrm{Cu}^{+}$的实时检测. 研究表明, 细胞 即使面临严重缺铜的环境中仍可通过调节达到线粒体 内铜含量的稳定.

为了提高一价铜离子探针 on/off 比值, Taki 等 ${ }^{[49]}$ 设 计合成了探针 38. 该探针在中性环境中形成螺环结构 导致其菼光消失，与 $\mathrm{Cu}^{+}$反应后探针中的罗丹明螺环开 环使得荧光恢复. 探针 38 的一大优势是可以通过更改 细胞器定位基团(例如 TPP 换为其他细胞器定位基团)而 达到对不同亚细胞层次一价铜离子定位检测的目的，这 就使得这种探针有能力成为阐明铜离子分布和新陈代 谢过程的重要工具.

\section{5 检测氢离子的线粒体荧光探针}

线粒体 $\mathrm{pH}$ 值直接影响线粒体中发生的生物化学过 程，并在一定程度上表明了细胞是否处于正常的生理状 态. 线粒体荧光探针能够实现线粒体 $\mathrm{pH}$ 的动态分布和 区域变化的实时监测(图 9).
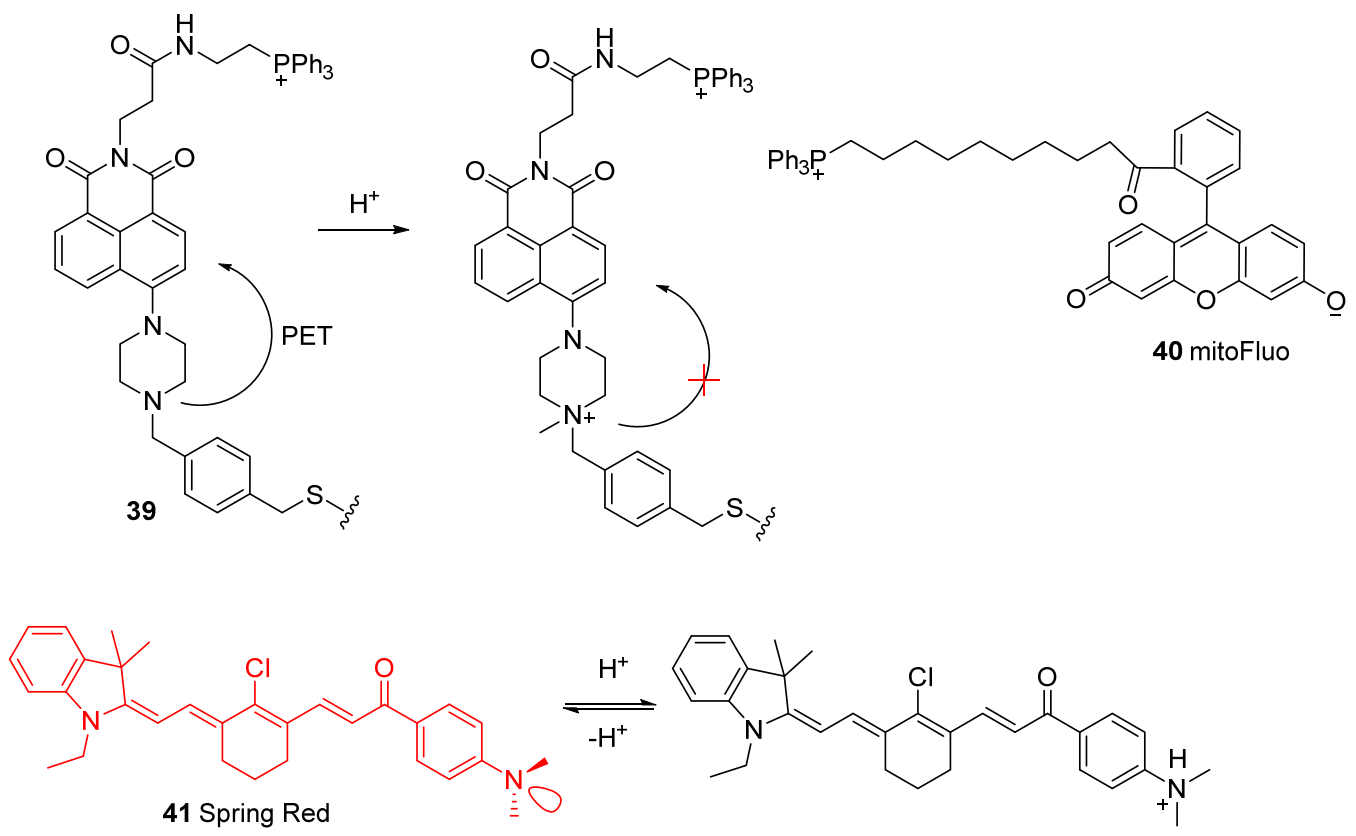

图 9 检测氢离子的线粒体荧光探针结构与响应原理

Figure 9 Targetable fluorescent probes in the mitochondria for detection of hydrogen ion: structures and responsing reactions 
基于 PET 原理, Kang 等 ${ }^{[50]}$ 设计合成了检测线粒体 $\mathrm{pH}$ 的关开型探针 39. 该探针质子化后 PET 禁阻被解除, 荧光恢复. 探针 39 可用于 HeLa 细胞内 $\mathrm{pH}$ 的实时检测 和成像，并可通过计算获得活细胞线粒体中的 $\mathrm{pH}$ 值. 探针 39 对线粒体酸化表现出令人满意的 off-on 荧光响 应, 有望应用于受损线粒体的监测, 以及相关的生理和 病理过程的研究.

Antonenko 小组 ${ }^{[51]}$ 报道的探针 $\mathbf{4 0}$ 不仅能够对质子 进行荧光检测与成像, 更是能够聚集在线粒体上帮助质 子通过线粒体膜并激发线粒体呼吸, 这使得该探针成为 定位于线粒体的解偶联剂, 有潜力成为治疗氧化应激相 关疾病的药物.

为实现活体 $\mathrm{pH}$ 检测与成像，唐波等 ${ }^{[52]}$ 设计合成了 可逆检测质子的新型近红外荧光探针 41. 该探针以长 共轭的半菁为近红外苂光团, 以三级胺为质子受体, 降 低了生物背景荧光的影响, 且不对称结构导致其 Stokes 位移较大. 探针 41 质子化后由于三级胺上的孤对电子 消失导致 $\mathrm{p}-\pi$ 共轭被破坏, 使其荧光减弱并发生蓝移, 能够可逆地检测线粒体 $\mathrm{pH}$ 变化. 探针 $\mathbf{4 1}$ 虽为近中性分 子, 该课题组认为其共振式结构中含有亲脂阳离子, 故 在膜电势驱动下能够定位于线粒体. 探针 41 还可用于 $\mathrm{HepG} 2$ 细胞、斑马鱼等活体的分子成像. 当用脂多糖 (LPS) 诱导的腹腔感染小鼠作为成像对象时, 探针荧光 强度降低，一定程度上说明受染组织中 $\mathrm{pH}$ 值有所降低.

\section{6 检测阴离子的线粒体荧光探针}

线粒体中阴离子虽然不如阳离子更受关注，但其作 用也是不可忽视的. 氟离子虽然在少量使用时益于牙齿 健康, 但在线粒体中富集则易导致代谢紊乱等一系列疾 病.

彭孝军等 ${ }^{[8]}$ 设计合成了高选择性检测氟离子(Eq. 4) 的线粒体苂光探针 42 . 氟离子与探针反应诱发了探针 结构中 $\mathrm{Si}-\mathrm{O}$ 键的断裂继而导致分子内环化反应的发 生, 使得探针的荧光从淬灭到恢复, 在 $485 \mathrm{~nm}$ 处发出强 的蓝绿色苂光, 并能在 COS-7、MCF-7 细胞中进行氟离 子分子成像.

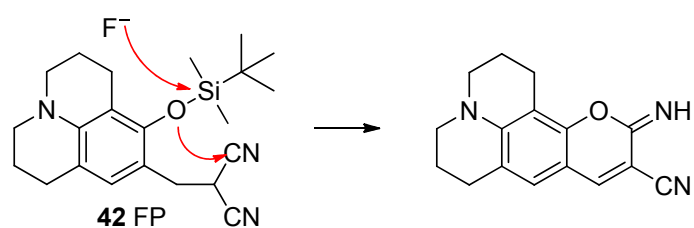

\section{7 总结与展望}

近几年基于有机小分子定位于线粒体的苂光探针 已经广泛用于 RSMs 的检测中, 其中双光子苂光探针应
用于苂光成像有激发波长红移至近红外、激发可定位、 观测时间更长等优点, 成为线粒体探针研究的热点. 值 得注意的是，在一些常见的苂光团上连接半菁单元，不 仅可作为线粒体定位基团，而且使最大吸收/发射波长 红移至近红外区，降低了生物背景苂光的影响，从而有 助于生物体无创成像; 该类探针已经逐渐成为新型线粒 体探针设计合成的发展方向. 在定位基团的选择与设计 上，线粒体探针虽然常以亲脂阳离子 TPP 和季铵盐作为 经典的线粒体定位基团，但是 TPP 的引入使得探针结构 复杂、分子量增大, 而季铵盐如吡啶盐易与线粒体上带 负电物质发生反应影响探针性质. 基于此, 一些中性线 粒体苂光探针的研究逐渐受到关注, 这类中性探针由于 不受线粒体膜电势的影响而使其具有独特的应用. 在应 用方面，线粒体探针作为 RSMs 检测成像的工具不断被 深入研究，利用线粒体探针进行疾病的前期诊断及后期 治疗预计将成为今后重要的发展方向之一.

\section{References}

[1] Cui, Y.; Long, J. G. In Mitochondrial Medicine and Health, Eds.: Liu, J. K.; Wang, X. M., Science Press, Beijing, 2012, Chapter 1.

[2] Pak, Y. L.; Swamy, K. M. K.; Yoon, J. Sensors 2015, 15, 24374.

[3] Carvalho, P. H. P. R.; Correa, J. R.; Guido, B. C.; Gatto, C. C.; Oliveira, H. C. B. D.; Soares, T. A.; Neto, B. A. D. Chem. Eur. J. 2014, 20, 15360 .

[4] Liu, X. L.; Du, X. J.; Dai, C. G.; Song, Q. H. J. Org. Chem. 2014, $79,9481$.

[5] Wei, L.; Yi, L.; Song, F. B.; Wei, C.; Wang, B. F.; Xi, Z. Sci. Rep. 2014, 4, 4521.

[6] Hirosawa, S.; Arai, S.; Takeoka, S. Chem. Commun. 2012, 48, 4845.

[7] Chen, J. H.; Liu, W. M.; Zhou, B. J.; Niu, G. L.; Zhang, H. Y.; Wu, J. S.; Wang, Y.; Ju, W. G.; Wang, P. F. J. Org. Chem. 2013, 78, 6121.

[8] Zhang, S. L.; Fan, J. L.; Zhang, S. Z.; Wang, X. W.; Du, J. J.; Peng, X. J. Chem. Commun. 2014, 50, 14021.

[9] Chan, J.; Dodani, S. C.; Chang, C. J. Nat. Chem. 2012, 4, 973

[10] (a) Ding, Y.; Tang, Y.; Zhu, W.; Xie, Y. Chem. Soc. Rev. 2015, 44, 1101 .

(b) Xie, Y.; Wei, P.; Li, X.; Hong T.; Zhang, K.; Furuta, H. J. Am. Chem. Soc., 2013, 135, 19119.

(c) Xie, Y.; Ding, Y.; Li, X.; Wang, C.; Hill, J. P.; Ariga, K.; Zhang, W.; Zhu, W. Chem. Commun. 2012, 48, 11513.

(d) Ding, Y.; Li, X.; Li, T.; Zhu, W.; Xie, Y. J. Org. Chem. 2013, 78, 5328.

(e) Chen, B.; Ding, Y.; Li, X.; Zhu, W.; Hill, J. P.; Ariga, K.; Xie, Y. Chem. Commun. 2013, 49, 10136.

[11] Neto, B. A. D.; Correa, J. R.; Silva, R. G. RSC Adv. 2013, 3, 5291.

[12] Leung, C. W. T.; Hong, Y. N.; Chen, S. J.; Zhao, E. G.; Lam, J. W. Y.; Tang, B. Z. J. Am. Chem. Soc. 2013, 135, 62.

[13] Zhao, N.; Li, M.; Yan, Y. L.; Lam, J. W. Y.; Zhang, Y. L.; Zhao, Y. S.; Wong, K. S.; Tang, B. Z. J. Mater. Chem. C 2013, 1, 4640.

[14] Zhao, N.; Chen, S.; Hong, Y.; Tang, B. Z. Chem. Commun. 2015, 51,13599

[15] Zhang, S.; Wu, T.; Fan, J. L.; Li, Z. Y.; Jiang, N.; Wang, J. Y.; Dou, B. R.; Sun, S. G.; Song, F. L.; Peng, X. J. Org. Biomol. Chem. 2013, 11,555 . 
[16] Jiang, N.; Fan, J. L.; Liu, T.; Cao, J. F.; Qiao, B.; Wang, J. Y.; Gao, P.; Peng, X. J. Chem. Commun. 2013, 49, 10620.

[17] Yang, W. G.; Chan, P. S.; Chan, M. S.; Li, K. F.; Lo, P. K.; Mak, N. K.; Cheah, K. W.; Wong, M. S. Chem. Commun. 2013, 49, 3428.

[18] Liu, X.; Sun, Y. M.; Zhang, Y. H.; Zhao, N.; Zhao, H. S.; Wang, G. C.; Yu, X. Q.; Liu, H. J. Fluoresc. 2011, 21, 497.

[19] Sarkar, A. R.; Heo, C. H.; Lee, H. W.; Park, K. H.; Suh, Y. H.; Kim, H. M. Anal. Chem. 2014, 86, 5638.

[20] Wu, S.; Cao, Q. Z.; Wang, X. L.; Cheng, K.; Cheng, Z. Chem. Commun. 2014, 50, 8919.

[21] Yuan, H(s).; Cho, H. O.; Chen, H. H.; Panagia, M.; Sosnovik, D. E.; Josephson, L. Chem. Commun. 2013, 49, 10361.

[22] Zhang, X. T.; Gu, Z. Y.; Liu, L. B.; Wang, S.; Xing, G. W. Chem Commun. 2015, 51, 8606.

[23] Liu, F.; Wu, J. F.; Cao, J. F.; Zhang, H.; Hu, M. M.; Sun, S. G.; Song, F. L.; Fan, J. L.; Wang, J. Y.; Peng, X. J. Analyst 2013, 138, 775.

[24] Xiao, H.; Xin, K.; Dou, H.; Yin, G.; Quan, Y.; Wang, R. Chem. Commun. 2015, 51, 1442

[25] Cheng, G. H.; Fan, J. L.; Sun, W.; Sui K.; Jin, X.; Wang, J. Y.; Peng, X. J. Analyst 2013, 138, 6091.

[26] Cheng, G. H.; Fan, J. L.; Sun, W.; Cao, J. F.; Hu, C.; Peng, X. J. Chem. Commun. 2014, 50, 1018.

[27] Hou, J. T.; Wu, M. Y.; Li, K.; Yang, J.; Yu, K. K.; Xie, Y. M.; Yu, X. Q. Chem. Commun. 2014, 50, 8640

[28] Dickinson, B. C.; Chang, C. J. J. Am. Chem. Soc. 2008, 130, 9638.

[29] Dickinson, B. C.; Lin, V. S.; Chang, C. J. Nat. Protoc. 2013, 8, 1249.

[30] Masanta, G.; Heo, C. H.; Lim, C. S.; Bae, S. K.; Cho, B. R.; Kim, H. M. Chem. Commun. 2012, 48, 3518.

[31] Xu, J.; Li, Q.; Guo, Y.; Yue, Y.; Shao, S. J. Biosens. Bioelectron. 2014, 56, 58 .

[32] Yu, H. B.; Zhang, X. F.; Xiao, Y.; Zou, W.; Wang, L. P.; Jin, L. J. Anal. Chem. 2013, 85, 7076.

[33] Sun, Y. Q.; Liu, J.; Zhang, H. X.; Huo, Y. Y.; Lv, X.; Shi, Y. W.; Guo, W. J. Am. Chem. Soc. 2014, 136, 12520.

[34] Li, P.; Zhang, W.; Li, K. X.; Liu, X.; Xiao, H. B.; Zhang, W.; Tang, B. Anal. Chem. 2013, 85, 9877.
[35] Xu, K. H.; Qiang M. M.; Gao, W.; Su, R. X.; Li, N.; Gao, Y.; Xie, Y. X.; Kong, F. P.; Tang, B. Chem. Sci. 2013, 4, 1079.

[36] Lim, C. S.; Masanta, G.; Kim, H. J.; Han, J. H.; Kim, H. M.; Cho, B. R. J. Am. Chem. Soc. 2011, 133, 11132.

[37] Lim, S. Y.; Hong, K. H.; Kim, D. I.; Kwon, H.; Kim, H. J. J. Am. Chem. Soc. 2014, 136, 7018.

[38] Liu, J.; Sun, Y. Q.; Zhang, H. X.; Huo, Y. Y.; Shi, Y. W.; Shi, H. P.; Guo, W. RSC Adv. 2014, 4, 64542.

[39] Han, C.; Yang, H.; Chen, M. ; Su, Q.; Feng, W.; Li, F. ACS Appl. Mater. Interfaces 2015, 7, 27968.

[40] Lee, M. H.; Han, J. H.; Lee, J. H.; Choi, H. G.; Kang, C.; Kim, J. S. J. Am. Chem. Soc. 2012, 134, 17314.

[41] Chen, Y. C.; Zhu, C. C.; Yang, Z. H.; Chen, J. J.; He, Y. F.; Jiao, Y.; He, W. J.; Qiu, L.; Cen, J. J.; Guo, Z. J. Angew. Chem., Int. Ed. 2013, 52, 1688.

[42] Bae, S. K.; Heo, C. H.; Choi, D. J.; Sen, D.; Joe, E. H.; Cho, B. R.; Kim, H. M. J. Am. Chem. Soc. 2013, 135, 9915.

[43] Yuan, L.; Zuo, Q. -P. Chem. Asian J. 2014, 9, 1544.

[44] Wang, X.; Sun, J.; Zhang, W. H.; Ma, X. X.; Lv, J. Z.; Tang, B. Chem. Sci. 2013, 4, 2551.

[45] Xue, L.; Li, G. P.; Yu, C. L.; Jiang, H. Chem. Eur. J. 2012, 18, 1050.

[46] Chyan, W.; Zhang, D. Y.; Lippard, S. J.; Radford, R. J. Proc. Natl. Acad. Sci. U. S. A. 2014, 111, 143.

[47] Masanta, G.; Lim, C. S.; Kim, H. J.; Han, J. H.; Kim, H. M.; Cho, B. R. J. Am. Chem. Soc. 2011, 133, 5698.

[48] Dodani, S. C.; Leary, S. C.; Cobine, P. A.; Winge D. R.; Chang, C. J. J. Am. Chem. Soc. 2011, 133, 8606.

[49] Taki, M.; Akaoka, K.; Mitsui, K.; Yamamoto, Y. Org. Biomol. Chem. 2014, 12, 4999.

[50] Lee, M. H.; Park, N.; Yi, C.; Han, J. H.; Hong, J. H.; Kim, K. P.; Kang, D. H.; Sessler. J. L.; Kang, C.; Kim, J. S. J. Am. Chem. Soc. 2014, 136, 14136.

[51] Denisov, S. S.; Kotova, E. A.; Plotnikov, E. Y.; Tikhonov, A. A.; Zorov, D. B.; Korshunova, G. A.; Antonenko, Y. N. Chem. Commun. 2014, 50, 15366.

[52] Li, P.; Xiao, H. B.; Cheng, Y. F.; Zhang, W.; Huang, F.; Zhang, W.; Wang, H.; Tang, B. Chem. Commun. 2014, 50, 7184. 